\title{
RIESGOS Y OPORTUNIDADES EN LA CRECIENTE DIGITALIZACIÓN FISCAL
}

\author{
María Amparo Grau Ruiz \\ Catedrática de Derecho Financiero y Tributario \\ Universidad Complutense de Madrid (España) \\ Directora de la Revista Técnica Tributaria
}

En los albores del proceso de reconversión digital para la recuperación económica en España, interesa hacer balance de algunos llamativos intentos de utilización de las herramientas tecnológicas disponibles para lograr una mejor gestión fiscal y el control del fraude en otros países de nuestro entorno.

Hasta cierto punto es inevitable que las técnicas novedosas y la forma de emplearlas, en sus inicios, adolezcan de fallos. Precisamente, por este motivo, su análisis resulta valioso para encauzar jurídicamente de manera adecuada posibles soluciones en un futuro próximo. Es pertinente prestarles aquí atención, en la medida en que su diseño puede favorecer o incluso mermar la justicia tributaria en aras de una mayor eficiencia.
Hoy en día, muchas instituciones, incluidas las Administraciones públicas, en sus procesos de modernización, han emprendido el camino hacia la detección y control del fraude dirigido por datos, buscando patrones en cantidades ingentes de información, a través de Departamentos cada vez más potentes. A raíz de estos nuevos métodos de trabajo, además del conocido problema de la fuga de datos sensibles -experiencia de

filtraciones (leaks) a la que no ha sido ajeno el mundo tributario-, se suscitan ahora nuevas controversias ${ }^{1}$.

La combinación de inteligencia artificial, datos masivos (big data) y procedimientos administrativos automatizados o robotizados -hasta con asistentes virtuales-, de no ser

1 La autora agradece a Paul Van der Smittte su opinión experta sobre este asunto y también su ayuda en la localización de materiales en neerlandés. Por un lado, conforme indica, la falta de personal, los recortes en el gasto público por la crisis y el volumen de trabajo hicieron que se usaran con ansias los datos disponibles. Por otro lado, el abundante intercambio automático de información contrasta con el relativamente escaso uso de la misma, conforme ha comprobado en el desarrollo de su tesis doctoral "Towards a more effective crossborder recovery of Tax Claims". 
acertada, puede entrañar graves riesgos para los contribuyentes. Efectivamente, empiezan a ponerse de relieve los primeros escollos en la incipiente transición digital fiscal. Una de las Administraciones tributarias europeas más dinámicas, la holandesa, ha experimentado recientemente un serio revés, al tratar de comprobar el correcto disfrute de beneficios fiscales mediante el uso de nuevas tecno$\operatorname{logías}^{2}$. Una regla de selección en el módulo del impuesto sobre la renta contenía el criterio de la nacionalidad, sin que hubiera un fundamento jurídico explícito para ello. En mayo de 2020, la Secretaría de Estado para beneficios y Aduanas informó a la Cámara de Representantes de que el Ministro de Hacienda anunció el cese de su uso el 15 de ese mismo mes ${ }^{3}$.
Detengámonos en el debate suscitado y en la sentencia recaída en este caso, que también despertó la atención del Relator Especial sobre la extrema pobreza y los derechos humanos de la $\mathrm{ONU}^{4}$.

Con una mirada crítica al papel otorgado a las tecnologías de la información y comunicación en la Administración tributaria y aduanera, en los Países Bajos se han denunciado abusos que han destruido financieramente a receptores de beneficios fiscales por cuidado de hijos, o hasta un posible componente racista en la actuación administrativa al poner el foco de los procedimientos en determinados inmigrantes. Todo ello, en la medida en que tecnológicamente era posible aplicar

2 Según NJCM y otros, la aplicación SyRI implica un enfoque de arrastre y no dirigido en el que se recogen datos personales con fines de investigación. Sostienen que SyRI es un sistema de seguimiento digital con el que se clasifica a los ciudadanos en perfiles de riesgo y en cuyo contexto el Estado utiliza el «aprendizaje profundo» y la minería de datos. En su opinión, SyRI es un sistema proactivo con una vinculación automatizada a gran escala, no estructurada y aleatoria de los archivos de grandes grupos de ciudadanos y un procesamiento secreto de datos personales. También aducen que la aplicación de SyRI entra en lo que se denomina en la literatura jurídica y en la práctica big data (apartado 6.45). Para fundamentar su posición, se apoyan en la «Opinión consultiva independiente sobre los efectos de la digitalización en las relaciones constitucionales», presentada al Gabinete por la División Consultiva del Consejo de Estado (apartado 6.46).

3 La segunda nacionalidad ya no se usaba en los modelos de riesgo centrales y las reglas de selección ya no proveían estos datos desde 31 de enero de 2015. No obstante, la selección podía hacerse por medio de consultas. La autoridad responsable de los datos personales está investigando si la Administración tributaria y aduanera puede haber procesado ilegalmente datos personales que revelen raza o etnia desde 2014 hasta la fecha. Antes de que se ajuste finalmente una liquidación provisional, siempre se realiza una comprobación por parte de un profesional (intervención humana). En el mes de mayo se les envió una «advertencia» de que la selección basada en la regla en cuestión no podía ser tratada. "Selectie naar nationaliteit bij voorlopige aanslag IB stopgezet" [Selección por nacionalidad interrumpida en caso de liquidación provisional de IB] Viditax, 12 de mayo de 2020. https://www.futd.nl/fiscaal-nieuws/14417/selectie-naar-nationaliteit-bij-voorlopige-aanslag-ib-stopgezet/ [último acceso: 10 de septiembre de 2020].

4 Como señala Philip Alston, Special rapporteur de Naciones Unidas, se trata del primer procedimiento que utiliza como fundamento jurídico el incumplimiento de un derecho humano para limitar el uso de determinadas tecnologías. Informe de amicus curiae en el caso de NJCM c.s./De Staat der Nederlanden (SyRI): Implicaciones del uso de las tecnologías digitales en los estados de bienestar, 26 de septiembre de 2019. https://www.ohchr.org/Documents/Issues/Poverty/Amicusfinalversionsigned.pdf y la Respuesta del Gobierno de los Países Bajos al caso SyRI, 29 de octubre de 2019 https:// www.ohchr.org/Documents/Issues/Poverty/20191029_Reply\%20NL\%20Govt_SyRI.pdf acceso: 10 de septiembre de 2020]. 
filtros para detectar a contribuyentes que tuvieran una segunda nacionalidad, careciendo de suficiente cobertura normativa tal modo de proceder ${ }^{5}$.

En nuestros días, el uso de perfiles a partir del análisis de datos es una técnica habitualmente empleada por las empresas. Se usa a gran escala y suele ser más eficaz que los muestreos aleatorios. Ahora bien, en el actuar administrativo, la forma en que se elabora y aplica un perfil de riesgo puede marcar la diferencia, cuando se tienen indebidamente en cuenta algunas características (por ejemplo, la edad, el género o la religión) que pueden llevar a una discriminación injustificada. Es especialmente importante la cuidadosa supervisión de la autoridad encargada de la protección de datos personales ${ }^{6}$.

La investigación de la autoridad de datos personales holandesa pone de relieve que el Departamento beneficios de la Administración tributaria no debería haber procesado la doble nacionalidad de los solicitantes del beneficio por cuidado de hijos. Estas operaciones de tratamiento de datos fueron ilegítimas, discriminatorias y violaron la legislación aplicable de protección de datos. En enero de 2014 dichos datos debieron haberse borrado, ya que con posterioridad a esa fecha dejaron de ser relevantes en la valoración de la concesión del beneficio en cuestión. Sin embargo, se guardaron y se usaron. Además, se emplearon para combatir el fraude organizado, cuando estos datos no eran necesarios para este fin. El sistema utilizaba la nacionalidad como indicador automático de ciertas operaciones de riesgo, sin que tampoco fuera necesario para este propósito. En definitiva, se hizo una distinción injustificada sobre la base de la nacionalidad, discriminatoria y prohibida.

Es ciertamente preocupante el almacenamiento y el uso de datos, a gran escala, por largos períodos de tiempo, por lo que deben extremarse las precauciones al respecto. Tras concederse al Ministro de Hacienda holandés el derecho a responder oficialmente a la investigación, en el otoño de 2020 puede que la autoridad de protección de datos anuncie una

5 Más allá del perfil étnico de un solo oficial investigador -aparentemente incompetente-, o una desafortunada coincidencia en un grupo arbitrario. René Veldwijk, «Data, ICT en de wantoestanden bij de Belastingdienst» [Datos, TIC y los abusos de la Administración Tributaria y Aduanera], Computable, 12 de julio 2020, p. 21. https://www.computable.nl/artikel/opinie/overheid/ 6957932/1509029/data-ict-en-de-wantoestanden-bij-de-belastingdienst.html [último acceso: 10 de septiembre de 2020].

6 La corrupción en los servicios públicos en ocasiones deja entrever prejuicios. Mieke Bean, «Over nut en nadeel van profileren voor de belastingdienst - Mag de overheid gebruik maken van risicoprofielen op basis van data-analyse?» [Sobre la utilidad y desventajas del perfilado para las autoridades tributarias - ¿Puede usar el gobierno perfiles de riesgo basados en el análisis de datos?, Trouw Letter en Geest, 30 de mayo de 2020. https://ris.utwente.nl/ws/files/206452459/ Boon_Trouw_May_25_2020.pdf [último acceso: 10 de septiembre de 2020]. 
posible sanción ${ }^{7}$. Por el momento, ya se ha eliminado la segunda nacionalidad de los modelos de riesgo y las reglas de selección. La primera nacionalidad sólo se incluye si existe una base jurídica para ello y si es necesaria para cumplir los deberes asignados ${ }^{8}$.

Es previsible que la opción por el trabajo administrativo orientado por decisiones a partir del tratamiento de datos siga afianzándose y se convierta en la norma en muchos países. En España, similares planteamientos se recogen expresamente en el ámbito estatal en las directrices generales del Plan Anual de Control Tributario y Aduanero de la AEAT publicadas en 2020: «Con el apoyo de las nuevas tecnologías se está procediendo de forma paulatina a una mejora de los sistemas de análisis de la información que permiten la ejecución de procesos de contraste de datos, utilizando tecnología big data, los cuales facilitan la detección temprana de conductas graves de defraudación, tales como las presuntas cantidades dejadas de ingresar y la presencia de economía sumergida, en la que el IVA tiene una especial relevancia» ${ }^{9}$. Del mismo modo, resuenan en el marco autonómico. Por ejemplo, en los criterios generales del Plan de control tributario de la Agencia Tributaria de Cataluña para el año 2020: «Refuerzo del modelo de gobernanza de los datos tributarios (big data) con la finalidad de mejorar la eficacia y la eficiencia en la lucha contra el fraude. Esta medida incluye mejorar la explotación de la tecnología para la gestión de datos externos, la detección y delimitación de patrones de fraude fiscal mediante la utilización de herramientas de analítica de datos, el diseño de tratamientos específicos efectuados para luchar contra el fraude y el

7 A finales de 2019 se pidió a la autoridad de protección de datos holandesa que investigara en 2020 la forma en que se crearon los modelos de riesgo, las normas de selección, los análisis de datos y las consultas en la Administración de Impuestos y Aduanas (incluidos los recargos), para determinar qué salvaguardias se aplican y cómo se prueban, contando con la participación del Oficial de Privacidad de la Administración de Impuestos y Aduanas y el Oficial de Protección de Datos del Ministerio de Finanzas. «Werkwijze Belastingdienst in strijd met de wet en discriminerend» [Método de trabajo de la Administración Tributaria y Aduanera contrario a Derecho y discriminatorio], Press release, 17 de julio de 2020. https://autoriteitpersoonsgegevens.nl/nl/nieuws/werkwijzebelastingdienst-strijd-met-de-wet-en-discriminerend [último acceso: 10 de septiembre de 2020].

8 Esto puede incluir casos concretos sobre salarios. Un «modelo de riesgo» estima la probabilidad de una corrección de, por ejemplo, las declaraciones, basándose en un conjunto de variables que explican lo mejor posible las correcciones de las declaraciones históricas. Las «reglas de selección» que forman parte de un módulo de selección se determinan sobre la base de los conocimientos y la experiencia del personal de la Administración tributaria y Aduanas. Ello puede incluir conocimientos profesionales, conocimiento de la legislación y los reglamentos o experiencia de años anteriores. Estas reglas de selección se utilizan para comprobar determinadas partes de la declaración de impuestos, por ejemplo, si un elemento específico de la deducción supera un determinado importe estándar. Las «consultas» son una recopilación de datos de múltiples fuentes de datos. En el caso de que no exista un fundamento jurídico explícito, se ha eliminado la nacionalidad de los datos personales de todas las consultas en uso o de las consultas antiguas. Carta del Secretario de Estado de Finanzas - Recargos y Aduanas de fecha 28 de abril de 2020, Características 2020-82842, www.rijksoverheid.nl.

9 En particular, para el control de actividades económicas. Resolución de 21 de enero de 2020, de la Dirección General de la AEAT, por la que se aprueban las directrices generales del Plan Anual de Control Tributario y Aduanero (BOE de 28 de enero de 2020). 
refuerzo en la detección y selección de riesgos de incumplimiento tributario» ${ }^{10}$.

En este contexto, debe subrayarse que no es lo mismo un funcionario de la administración tributaria que toma decisiones apoyándose en datos y programas ejecutados informáticamente, que un algoritmo que adopte decisiones y éstas se ejecuten por un funcionario. Es fundamental la constante supervisión del desarrollo tecnológico en este campo, para advertir en qué momento pivota el procedimiento de expertos tributarios, buenos conocedores de las reglas de interpretación normativa, a programadores que hacen uso de inteligencia artificial -salvo que se cuente con profesionales que reúnan ambas competencias-.. En todo caso, la tecnología habrá de ser pautada conforme a parámetros que han reflejar la legalidad vigente. Y este asunto, es bastante más complejo de lo que a simple vista parece, como ponen de relieve los notorios esfuerzos en materia de innovación en la OCDE y una propuesta experimental en Francia a la hora de traducir las normas a códigos de programación, sobre todo, en materia tributaria ${ }^{11}$.

Obviamente, los patrones que detecte un determinado software a partir de conjuntos de datos pueden ser útiles y merecen ser aprovechados por la Administración pública. Pero, al mismo tiempo, se reivindica que deben ser conocidos y los ciudadanos

10 Concretamente, entre las actuaciones de prevención del fraude tributario. Resolución VEH/ 522/2020, de 7 de febrero, de la Comunidad Autónoma de Cataluña, por la que se da publicidad a los criterios generales del Plan de control tributario de la Agencia Tributaria de Cataluña para el año 2020 (DOGC de 2 de marzo de 2020). También, anteriormente, en la Hacienda foral: «b) Dentro de los desarrollos tecnológicos dirigidos a evitar la realización de conductas fraudulentas, se enmarcan los proyectos Big Data en marcha: • Proyecto relaciones entre contribuyentes. En 2018 se realizó un proyecto piloto en el que se implementó una infraestructura de Big Data para explotar los datos de relaciones entre contribuyentes existentes en las bases de datos de Hacienda. Este año 2019 se da continuidad al piloto incorporando mejoras y convirtiéndolo en una aplicación en explotación: -Preparación de la infraestructura definitiva de Big Data, basada en la del piloto. - Automatización y mejora de los procesos de carga de datos. - Mejora en la calidad de los datos incorporados. Limpieza de inconsistencias y duplicidades. - Nuevo sistema de visualización y explotación de los datos. - Proyecto análisis de riesgo en base a información del SII. Es un proyecto piloto que permite hacer una primera aproximación analítica a los datos que se han ido recogiendo desde la implantación del sistema de Suministro Inmediato de Información. El proyecto va a utilizar la misma infraestructura de Big Data definida para el proyecto de relaciones. El objetivo es analizar los datos del SII, tanto entre ellos mismos como relacionados con las autoliquidaciones de IVA y otros datos fiscales de interés. Se han definido un conjunto de casos de análisis, cada uno de los cuales generará unos informes alertando de situaciones de riesgo de fraude». Orden Foral 135/2019, de 4 de marzo, del Territorio Histórico de Guipúzcoa, relativa al Plan de Comprobación Tributaria para el año 2019, que incluye como anexo el Plan de Inspección del ejercicio 2019, así como los criterios generales que lo informan (BOG de 11 de marzo de 2019).

11 En 2019, el Instituto Nacional de Investigación en Informática de Francia (Inria) lanzó una iniciativa centrada en el desarrollo de un nuevo lenguaje para reflejar las reglas como código de programación: Catala. Busca lograr la equivalencia semántica con la ley y traduce cada línea de un texto legislativo como un fragmento de código. Esto permite que los programas sean fácilmente verificados y validados. También comprende un compilador que mejora la interoperabilidad con otros lenguajes. https://catala-lang.org [último acceso: 10 de septiembre de 2020].

A finales de 2019, en la OCDE, en su observatorio de la innovación en el sector público (OPSI, por sus siglas en inglés) se comenzó a trabajar en el Manual de Innovación centrado en el concepto de Reglas como Código, diseñadas «para el consumo humano y de las máquinas» en la era digital para que el gobierno sea verdaderamente transparente y efectivo. Borrador «Descifrando el Código»: Creación de reglas para humanos y máquinas» (Cracking the Code: Rulemaking for humans and machines) https://oecd-opsi.org/seeking-your-feedback-on-draft-rules-as-code-primer/[último acceso: 10 de septiembre de 2020]. 
merecen que se les puedan ser explicados, salvaguardando las garantías de confidencialidad. Al menos, que puedan comunicarlos los empleados y verificarlos los supervisores. Para una buena relación entre el Gobierno y los ciudadanos, es fundamental poder controlar la procedencia y calidad de los datos en los que se basan las decisiones administrativas, sin que la oscuridad o complejidad sirvan como excusa tras la que puedan esconderse quienes tengan la tentación de cometer posibles arbitrariedades o corruptelas. Como bien se ha dicho, de lo contrario, la administración tributaria suministrará programas (con errores), datos (sesgados) y, en el peor de los casos, a través de empleados expertos en tecnologías de la información y comunicación que se habrán equivocado ${ }^{12}$.

12 Desde luego, conviene afrontar los problemas cuanto antes, pues la recolección y el uso de datos va a ganar peso exponencialmente. Cada vez hay más fuentes de información disponibles. Por ejemplo, sobre movilidad: cámaras en carreteras, aparcamientos, vuelos, etc.; o sobre consumos: de energía, o telefónicos. En España, la Dirección General de la Agencia Estatal de Administración Tributaria cuenta con datos procedentes del mismo interesado, de otras Administraciones Públicas, de otras personas físicas distintas al interesado, de entidades privadas, de registros públicos y de fuentes accesibles al público. Realiza tratamientos asociados a la gestión sistema tributario estatal y aduanero, y otros cuya finalidad y la información detallada se describe en cada una de las actividades de tratamiento que realiza la AEAT, accesibles en el registro. https:// www.agenciatributaria.es/AEAT.internet/Inicio/Ayuda/Modelos__Procedimientos_y_Servicios/

Ayuda_P_FZO8__Tratamiento_de_datos_personales/Informacion_general/

_Ayuda_Informacion_al_interesado_sobre_proteccion_de_datos/

5__Registro_de_las_actividades_de_tratamiento/

5__Registro_de_las_actividades_de_tratamiento.html [último acceso: 10 de septiembre de 2020].

Queda lejos en el tiempo la época en la que se decidía únicamente con la información aportada por el contribuyente y hasta se cuenta con la información que puedan proporcionar plataformas. «Las características de la economía de las plataformas digitales complican en gran medida la trazabilidad y la detección de los hechos imponibles por parte de las administraciones tributarias. El problema se agrava especialmente cuando estas operaciones se realizan a través de operadores de plataformas digitales establecidos en otra jurisdicción. El hecho de que los vendedores no comuniquen las rentas obtenidas a cambio de la prestación de servicios o la venta de bienes a través de plataformas digitales conlleva un déficit en los ingresos fiscales de los Estados miembros. También otorga una ventaja a esos vendedores en comparación con los que no operan en plataformas digitales»(p.2). «En pro de la eficiencia, se anima a los Estados miembros a que permitan la notificación digital y garanticen la interoperabilidad de los sistemas así como a nivel de los datos entre las plataformas digitales y las administraciones fiscales en la medida de lo posible» (p.9). «La presente Directiva respeta los derechos fundamentales y observa los principios reconocidos, en particular, por la Carta de los Derechos Fundamentales de la Unión Europea. En particular, el conjunto de elementos de datos que deben transmitirse a las administraciones tributarias se definen de tal manera que recaben los datos mínimos necesarios para detectar los incumplimientos debidos a la escasez de comunicación de información o a la no comunicación de la misma, de acuerdo con las obligaciones establecidas en el RGPD» (p.10). Comisión Europea (Dirección General de Fiscalidad y Unión Aduanera), Propuesta de Directiva del Consejo por la que se modifica la Directiva 2011/16/UE relativa a la cooperación administrativa en el ámbito de la fiscalidad. COM/2020/314 final, Bruselas, de 15 de julio de 2020 2020/0148 (CNS), https://eur-lex.europa.eu/legal-content/ES/TXT/DOC/?uri=CELEX: 52020PC0314\&qid=1599770668228\&from=ES [último acceso: 10 de septiembre de 2020]. No está de más traer a colación que hace tan solo unos meses la Sala de lo Contencioso-Administrativo del Tribunal Supremo en la Sentencia 1106/2020, de 23 de julio de 2020, Rec. n. ${ }^{\circ}$ 80/2018, ha decidido sobre la anulación de la obligación de informar sobre la cesión de uso de viviendas con fines turísticos. De acuerdo con la STJUE de 19 de diciembre de 2019, C-390/18 recaída en el asunto Airbnb Ireland, se desprende que una norma como el art. 54 ter REGAT debía haber sido notificada bajo la Directiva 1535/2015 y que su falta de notificación conlleva su invalidez. 
Todo este movimiento se ha visto impulsado por la Sentencia de un Tribunal holandés en La Haya, de 5 de febrero de $2020^{13}$, que declara contrario al derecho al respeto a la vida privada previsto en el artículo 8.2 del Convenio Europeo para la Protección de los Derechos Humanos y de las Libertades Fundamentales ${ }^{14}$ (en adelante, CEDH), el uso de un sistema algorítmico de indicación de riesgos (System Risk Indication o SyRI, por sus siglas en inglés) para predecir la probabilidad de que solicitantes de beneficios estatales defraudaran tanto en sus contribuciones a la Seguridad Social como en el pago de impuestos. Recuerda este Tribunal que ha de buscarse el equilibrio entre las ventajas asociadas al uso de las nuevas tecnologías y las interferencias que pueden causarse en este derecho.
Como se dice en el apartado 6.7 de la citada Sentencia, la legislación sobre SyRI no cumple el requisito de que la injerencia en el ejercicio de tal derecho en una sociedad democrática sea necesaria, proporcionada y subsidiaria. Opina el Tribunal que no logra un equilibrio justo entre el interés social al que sirve y la violación del derecho. Toma en consideración los principios fundamentales sobre la protección de datos en el Derecho de la Unión (concretamente los principios de transparencia, limitación de la finalidad y minimización de los datos) ${ }^{15}$, pues entiende que el nivel mínimo de protección del derecho al respeto de la vida privada -incluida la protección de los datos personalesen virtud del CEDH, no es menos amplio que la protección de datos que ofrecen la Carta de los Derechos

13 ECLI:NL:RBDHA:2020:1878. Puede consultarse la versión en inglés: The Hague District Court, Commerce Team, Case No.: C/09/550982 (cause list No.: HA ZA 18-388) Judgment of 5 February 2020, Nederlands Juristen Comité Voor De Mensenrechten, Stichting Platform Bescherming Burgerrechten, Stichting Privacy First, Stichting Koepel Van Dbc-Vrije Praktijken, Landelijke Cliëntenraad [Claimant Sub 6 And 7] and Federatie Nederlandse Vakbeweging versus the State of The Netherlands. https:// uitspraken.rechtspraak.nl/inziendocument?id=ECLI:NL:RBDHA:2020:1878 [último acceso: 10 de septiembre de 2020].

14 https://www.echr.coe.int/documents/convention_spa.pdf [último acceso: 10 de septiembre de 2020].

15 NJCM y otros añadieron en su argumentación referencias a los artículos 7 y 8 de la Carta de los Derechos Fundamentales de la Unión Europea y/o el artículo 17 del Pacto Internacional de Derechos Civiles y Políticos y/o el artículo 6 y/o el artículo 13 del CEDH; y/o los artículos 5, 6, 13, 14, 22 y/o 28 del RGPD. Conforme al artículo 22 del Reglamento General de Protección de Datos: «todo interesado tendrá derecho a no ser objeto de una decisión basada únicamente en el tratamiento automatizado, incluida la elaboración de perfiles, que produzca efectos jurídicos en él o le afecte significativamente de modo similar». No obstante, el Tribunal no aclara el contenido del derecho a opt out."Derechos humanos en un mundo de algoritmos: la sentencia histórica que ata en corto la implantación de modelos opacos", The technolawgist, 12 de febrero de 2020 https://www.thetechnolawgist.com/ 2020/02/12/derechos-humanos-en-un-mundo-de-algoritmos-la-sentencia-historica-que-ata-en-cortola-implantacion-de-modelos-opacos/ [último acceso: 10 de septiembre de 2020]. 
Fundamentales y el RGPD (apartado 6.41).

Además, dado que la legislación relativa a la aplicación de SyRI no es lo bastante transparente y verificable, declara que el artículo 65 de la Ley SUWI ${ }^{16}$ y el capítulo 5a del Decreto SUWI no tienen ningún efecto vinculante, por ser contrarios al párrafo 2 del artículo 8 del CEDH. En definitiva, la legislación debe ofrecer un marco eficaz que permita sopesar los intereses en juego de manera transparente y verificable. Todas las personas deben poder tener la expectativa razonable de que su vida privada se respeta suficientemente (apartado 6.6 de la referida sentencia).

El Tribunal tiene en cuenta que parte del derecho a la protección de los datos personales es el derecho de toda persona a estar razonablemente en condiciones de hacer un seguimiento de sus datos personales y a ser informada sobre su tratamiento. Aunque el inicio de un proyecto SyRI se publica en el Boletín Oficial, un informe de riesgo puede conservarse en el registro durante dos años, sin que el interesado lo sepa (apartado 6.60). Añade que el modelo de riesgos que se utiliza actualmente y los indicadores son «secretos». Esto se aplica también a los datos utilizados en un proyecto SyRI concreto ${ }^{17}$.

Pese a que uno de los propósitos de estos proyectos fuera contribuir a la mejora de las condiciones de vida en los vecindarios, la falta de transparencia del algoritmo ${ }^{18}$ y su uso sesgado en barrios donde viven personas pertenecientes a minorías puede perpetuar discriminaciones a partir de series históricas de datos y amplificar prejuicios sociales. Al respecto, cabe recordar que el derecho al respeto de la vida privada

16 Wet structuur uitvoeringsorganisatie werk en inkomen (SUWI) Ley sobre la estructura de la organización administrativa del trabajo y los ingresos. Se pretendía una acción gubernamental integral en la prevención y la lucha contra el fraude fiscal y de la Seguridad Social y el incumplimiento de las leyes laborales. Los organismos participantes en una alianza de colaboración estaban obligados a facilitarse mutuamente la información necesaria, siendo controladores conjuntamente en el sentido del artículo 26 del RGPD (artículo 64, párrafo 3 de la Ley SUWI). Según la exposición de motivos del Decreto SUWI, los indicadores y el modelo de riesgo a aplicar debían identificarse claramente y sin esta especificación la vinculación de los registros de datos podría dar lugar a una «expedición de pesca» e incluso a arbitrariedades. Según el Ministro, este método hacía justicia al principio de «seleccionar antes de recoger». Según el Decreto SUWI, un indicador es cualquier información que hace plausible la presencia de una circunstancia particular. Por modelo de riesgos se entiende un modelo que consta de indicadores predeterminados y que indica si existe un mayor riesgo de utilización ilícita de fondos y planes gubernamentales.

17 El modelo de riesgos, los indicadores y los datos que se han procesado concretamente no son públicos, ni son conocidos por los interesados. La legislación sobre SyRI no prevé la obligación de informar a las personas de que sus datos han sido procesados. Tampoco existe la obligación de informar a los interesados individualmente de que se ha presentado un informe de riesgo, pese a que tiene un efecto significativo en la vida privada de la persona a la que pertenece el informe» (apartado 6.65).

18 La transparencia figura entre los principios éticos publicados por la Comisión Europea en abril de 2019, así como entre los diez principios básicos en materia de inteligencia artificial para su uso por las agencias federales en EE.UU. 
en el contexto del tratamiento de datos se refiere al derecho a la igualdad de trato en casos iguales y al derecho a la protección contra la discriminación, los estereotipos y la estigmatización (apartado 6.24).

De la misma forma que se auditan las cuentas de empresas y entes públicos, se propone la realización de auditorías de algoritmos para la detección de sesgos ${ }^{19}$. Por su parte, la Secretaría de Estado indicó que no puede compartir los modelos de riesgo y las reglas de selección con por la naturaleza confidencial de esta información. Ofreció a los parlamentarios, en cambio, una sesión informativa técnica sobre el funcionamiento y el proceso de desarrollo de los modelos de riesgo y los módulos de selección ${ }^{20}$.

Es lógico que se utilicen las opciones digitales para vincular archivos y analizar datos con la ayuda de algoritmos, y también las posibilidades de las autoridades de intercambiar datos para cumplir su deber de prevenir y combatir el fraude, cuando ello redunda en mayor bienestar económico por verificarse la exactitud y la integridad de los datos por los que los ciudadanos reciben ciertos beneficios. Sin embargo, la existencia de protección legal adecuada de la privacidad contribuye a la confianza de los ciudadanos en el gobierno, tanto como lo hacen la prevención y la lucha contra el fraude. Sin una protección suficiente del derecho al respeto de la vida privada, es poco probable que los ciudadanos estén dispuestos a proporcionar datos. Por eso, cabe destacar los pasos que -hasta en las antípodas- se dan para el uso responsable de avances tecnológicos 21 .

19 «En Estados Unidos a mediados de 2019 los senadores Cory Booker y Ron Wyden presentaron la Ley de responsabilidad algorítmica, un proyecto de ley que requeriría los algoritmos utilizados por compañías que ganan más de 50 millones de dólares al año o que retengan información de al menos 1 millón de usuarios para evaluar sesgos. Lo interesante de esta medida es que la auditoría exige un análisis en tres partes: (i) del proceso de diseño del algoritmo en si mismo; (ii) de los datos utilizados para entrenarlo y para obtener los resultados; y (iii) de los resultados mismos». "Algorithmic Accountability Act: así se está regulando el futuro del big data y los algoritmos en Estados Unidos", The technolawgist, 3 de junio de 2019. https://www.thetechnolawgist.com/2019/06/03/algorithmicaccountability-act-regulando-el-futuro-big-data-los-algoritmos-estados-unidos/ [último acceso: 10 de septiembre de 2020].

20 Documentos parlamentarios II 2019/20, 31066, no. 538.

21 La Algorithm Charter for Aotearoa New Zealand ha sido rubricada por 25 organismos públicos, demostrando el compromiso global del gobierno y sector público en un tema tan relevante para la seguridad y confianza de los ciudadanos: la protección de sus datos. Esta Carta será revisada periódicamente, sin frenar los avances tecnológicos, permitiendo evaluar su buena incorporación, adaptación y utilización - sin sesgos ni opacidades-. «Nueva Zelanda, un ejemplo de transparencia y responsabilidad en el uso de los datos por parte del sector público», The Technolawgist 6 de agosto de $2020 \quad$ https://www.thetechnolawgist.com/2020/08/06/nueva-zelanda-un-ejemplo-detransparencia-y-responsabilidad-en-el-uso-de-los-datos-por-parte-del-sector-publico/ El texto completo en inglés de esta Carta se encuentra publicado en https://data.govt.nz/assets/data-ethics/ algorithm/Algorithm-Charter-2020_Final-English-1.pdf [último acceso: 10 de septiembre de 2020]. 